\title{
A Machine Learning approach to predict Healthcare-Associated Infections at Intensive Care Unit admission: findings from the SPIN-UTI project
}

\author{
Martina Barchitta ${ }^{1,2}$, Andrea Maugeri ${ }^{1,2}$, Giuliana Favara ${ }^{1}$, Paolo Riela $^{3}$, Giovanni Gallo ${ }^{3}$, Ida Mura ${ }^{2,4}$, Antonella \\ Agodi $^{1,2^{*}}$, on behalf of the SPIN-UTI network ${ }^{\#}$ \\ ${ }^{1}$ Department of Medical and Surgical Sciences and Advanced Technologies "GF Ingrassia", University of \\ Catania, Catania, Italy \\ ${ }^{2}$ GISIO-SItI - Italian Study Group of Hospital Hygiene - Italian Society of Hygiene, Preventive Medicine and \\ Public Health, Italy \\ ${ }^{3}$ Department of Mathematics and Informatics, University of Catania, Catania, Italy \\ ${ }^{4}$ Department of Biomedical Sciences, University of Sassari, Sassari, Italy
}

*Corresponding author: Antonella Agodi, Department of Medical and Surgical Sciences and Advanced

Technologies "GF Ingrassia", University of Catania, Catania, Italy. Via S. Sofia, 87 - 95123 Catania, Italy.

agodia@unict.it

Running title: A Machine Learning approach to predict Healthcare-Associated Infections

\begin{abstract}
Purpose

To evaluate the performance of the SAPS II score for Healthcare Associated Infections (HAIs) risk prediction in Intensive Care Units (ICUs) using both traditional statistical and machine learning approaches.

\section{Methods}

We used data of 7827 patients enrolled from the "Italian Nosocomial Infections Surveillance in Intensive Care Units" (SPIN-UTI) project, with complete information at ICU admission. The Support Vector Machines (SVM) algorithm with Gaussian Kernel was applied to classify patients according to sex, patient's origin, non-surgical treatment for acute coronary disease, surgical intervention, SAPS II score at admission, presence of invasive devices at ICU admission, trauma, impaired immunity, antibiotic therapy in 48 hours before ICU admission.

\section{Results}

Traditional statistical approach showed that the performance of SAPS II score for predicting the risk of HAIs provides a ROC (Receiver Operating Characteristics) curve with an AUC (Area Under the Curve) of 0.612 $(95 \%$ Confidence Interval $=0.60-0.63 ; \mathrm{p}<0.001)$ and an accuracy of $56 \%$. Considering SAPS II score along with other characteristics at ICU admission, we found that the accuracy of the SVM classifier was $88 \%$ on the test set, with a ROC curve which provided an AUC of $0.90(95 \% \mathrm{CI}=0.88-0.91 ; \mathrm{p}<0.001)$. In line, the predictive ability was lower when considering the same SVM model, without accounting for the SAPS II score. Indeed, we estimated an accuracy of $78 \%$ and an AUC of $0.66(95 \% \mathrm{CI}=0.65-0.68 ; \mathrm{p}<0.001)$.
\end{abstract}

\section{Conclusions}

Our study suggested the SVM model as a possible tool to quickly predict patients at the highest risk of HAI at ICU admission.

Keywords: healthcare-associated infections; machine learning; intensive care unit; risk prediction 


\section{Introduction}

Healthcare Associated Infections (HAIs) are one of the major threats for public health worldwide, due to their significant impact on mortality, hospital stays, and assistance costs [1-3]. In particular, frequency of HAIs is higher among people staying in Intensive Care Units (ICUs), because they have more severe clinical conditions, they are often immune-compromised, and more likely to be intubated and catheterized than those staying in other hospital wards $[4,5]$. Furthermore, high antibiotic resistance rates have been reported together with increasing trends of resistant microorganisms, highlighting the need for continuous comprehensive strategies targeting not only the prudent use of antibiotics, but also infection control measures to control the epidemic spread of resistant isolates, especially in ICUs $[3,6]$.

As reported by the European Center for Disease Prevention and Control (ECDC), in 2017 on a total of approximately 143,000 patients staying in ICU, $8 \%$ presented at least one HAI on a given day. In line, among ICU-surveilled HAIs, pneumonia, bloodstream infection (BSI) and urinary tract infections (UTIs) accounted for $6 \%, 4 \%$ and $2 \%$, respectively [7].

Although HAIs depend on microorganisms' characteristics - such as infectivity, pathogenicity, modes of transmission - several patients' characteristics and the inappropriate use of invasive devices during the hospital stay represent some of the leading causes of HAIs in all the hospital wards, and especially in ICUs $[4,8]$. In the last decades, several prognostic scores have been developed in clinical practice to measure health conditions or illness severity of ICU patients. In particular, the Simplified Acute Physiology Score (SAPS) II represents the most widely used instrument for the prediction of prognosis, HAIs risk, sepsis and mortality [9-13]. This validated score is calculated considering twelve routine physiological variables collected during the first hours of ICU admission, not including the type admission [14, 15]. For these reasons, the identification of patients at higher risk of HAIs in ICU still remains a major challenge for public health, with so many healthcare professionals which have studied and continue to examine personal and clinical characteristics associated with HAI risk [16-21]. In this scenario, recent advances in statistical and mathematical approaches to automatically learn from a given dataset have made possible to identify patients or subgroups of patients which are more likely to be affected by HAI during their hospital stay [22-24] . Indeed, there is a strong need for reliable clinical tools that can guide patient management [25] by predicting the risk of HAIs and adverse associated outcomes, and thus reducing their burden on healthcare systems $[26,27]$.

Here, we aimed to identify and predict patients at risk of HAIs, according to their characteristics at ICU admission. To do that, we used data from the "Italian Nosocomial Infections Surveillance in Intensive Care Units" (SPIN-UTI) project, which was established by the Italian Study Group of Hospital Hygiene (GISIO) of the Italian Society of Hygiene, Preventive Medicine and Public Health (SItI) in 2006. The SPIN-UTI network, since then, has collected data related to approximately 20,000 patients, more than 4,300 infections and 5,300 microorganisms [16-21,28]. Our hypothesis is that machine learning algorithms could enrich conventional statistical approaches, especially in terms of prediction of ICU prognosis, clinical deterioration and risk assessment [29]. Accordingly, the current study first evaluates the performance of the SAPS II score for HAI's risk prediction in ICUs using a traditional statistical method. Next, we applied a Support Vector Machines (SVM) algorithm, considering SAPS II score in combination with additional features at ICU admission, in order to distinguish non-infected patients from those who were diagnosed with at least one HAIs during their ICU stay. 


\section{Methods}

Study design and data collection

In the current study, we used data collected during the seven editions of the SPIN-UTI project according to the European Centre for Disease Control and Prevention (ECDC) protocol [30]. From 2006 to 2019, the SPIN-UTI project surveyed 20060 patients staying in ICUs for more than 2 days, recording data at patient, ICU and hospital levels during their stay in ICU. Study design, protocols and full details on data collection were described elsewhere $[16-21,28]$. The original dataset contained only $39 \%$ of patients $(n=7827)$ with a complete assessment of variables considered in our study (Supplementary Figure 1). Since machine learning approaches require large data sets for training, we built a novel training data set made of recovered and synthetics data to tune the learning algorithms, together with a test set composed only by real data of patients with a complete assessment of the following variables at ICU admission: sex (dichotomous), patient's origin (categorical: other ward/healthcare facility, community), non-surgical treatment for acute coronary disease (dichotomous), surgical intervention (dichotomous), SAPS II score at admission (continuous), presence of invasive devices at ICU admission (three dichotomous variables for urinary catheter, intubation and central venous catheter, respectively), trauma (dichotomous), impaired immunity (dichotomous), antibiotic therapy in 48 hours before ICU admission (dichotomous). Methods for data imputation and balancing are fully described in the Supplementary Materials.

\section{Training and Test Set composition and comparison}

The training set is made by recovered $(\mathrm{n}=7758)$ and synthetics records $(\mathrm{n}=2544)$, while the test set includes 7827 real data. The distribution of infected and non-infected patients between the training and test sets is summarized in Supplementary Table 1. To evaluate the goodness of the training set records, we compared the distributions of each single variable with those of the test set to assess that the training data are compliant with the real data. As reported by Supplementary Figure 2, the variables SAPS II score and age follows the same distribution of the training and test sets. Likewise, Supplementary Figures $\mathbf{3}$ and $\mathbf{4}$ show that the distributions of categorical variables are similar between training and test sets.

\section{Learning model generation}

To improve the predicting performance of the model, a machine learning algorithm combining the SAPS II with additional variables collected at ICU admission (i.e. sex, patient's origin, non-surgical treatment for acute coronary disease, surgical intervention, presence of intubation, presence of urinary catheter, presence of central vascular catheter; trauma, impaired immunity, antibiotic therapy in 48 hours before ICU admission) was applied. Specifically, we chosen the Support Vector Machine (SVM) with Gaussian Kernel (RBF) as modeling tool. This model has been successfully used in several regression and classification studies, especially for binary classification problems. Our model classifies data finding the best hyperplane separating the points of the classes. The separating hyperplane found by the algorithm provides the largest margin between the two classes. The selection of a non-linear kernel function, in our case the Gaussian kernel, is useful to map data that are not originally linearly separable into a higher dimensional feature space where they are made linearly separable. It is worth mentioning that linear kernels are less time consuming than non-linear ones, but they provides less 
accuracy [31]. Data analyses were performed through Python and the SciPy stack. Full details on the computational methods are given in the Supplementary Material.

\section{Statistical Analysis}

Statistical analyses were performed using SPSS software (version 26.0, SPSS, Chicago, IL). The KolmogorovSmirnov test was used to check the normal distribution of continuous variables. Patients' characteristics were described using median and interquartile range (IQR) or percentage.

Comparisons between variables were analyzed by the Chi-squared test for categorical variables, while the Mann-Whitney $U$ test was used for continuous variables with skewed distribution. To test the accuracy of the SAPS II score in HAI's risk prediction along the range of possible values, we used ROC (Receiver Operating Characteristics) curve analysis. In particular, discrimination was assessed by calculating the area under the curve (AUC), with values ranging from 0.5 for no prediction to 1.0 for perfect prediction $[9,11,32,33]$. All statistical tests were two-sided, and p-values $<0.05$ were considered statistically significant.

\section{Results}

\section{Study population}

On a total of 20060 SPIN-UTI participants, the current analysis was performed on a subsample of 7827 patients (median age $=69$ years; $60.6 \%$ males) enrolled from 2006 to 2019 . The remaining 12233 participants $(61 \%)$ were excluded because of missing data on the assessment at ICU admission. In this subsample, patients coming from other wards/hospitals and reporting a surgical type of ICU admission were $73.9 \%$ and $52.4 \%$, respectively. In general, median SAPS II score at admission was $40(\mathrm{IQR}=28)$ and length of ICU stay was 5 days $(\mathrm{IQR}=10)$. Patients who reported trauma and impaired immunity were $3.4 \%$ and $8.6 \%$, respectively. With respect to medical treatments, $10.2 \%$ and $40.9 \%$ of patients underwent to non-surgical treatment for acute coronary disease or surgical intervention, while e 59\% patients were on antibiotic therapy. In particular, the presence of urinary catheter, intubation and central venous catheter was $77.5 \%, 59.8 \%$ and $41 \%$, respectively. Finally, we observed that percentage of ICU-acquired sepsis among patients enrolled was $6.1 \%$, whereas ICU mortality was $23.2 \%$.

\section{Characteristics of infected patients}

Overall, Table 1 also shows the comparison between infected $(n=1225 ; 15.7 \%)$ and non-infected patients $(n=$ $6602 ; 84.3 \%$ ) for characteristics at ICU admission. Infected patients were more likely to come from the community and to report a medical type of ICU admission than those non-infected. In particular, infected group consisted of patients who were more likely to report impaired immunity, also including more patients with trauma. This translated to higher SAPS II score among infected patients if compared with non-infected.

With respect to the presence of invasive devices, infected patients were also more likely to be intubated at ICU admission and less likely to be catheterized than those non-infected. As expected, infected patients exhibited higher length of ICU stay ( 20.0 days vs. 4.0 days; $p<0.001)$ compared to non-infected patients. In line with these findings, also mortality was higher in infected patients $(35.1 \%)$ than in those non-infected $(21.0 \% ; \mathrm{p}<0.001)$. No differences were evident for age, sex, non-surgical treatment for acute coronary disease, antibiotic therapy in 48 hours before ICU admission and presence of central venous catheter at ICU admission.- 
ROC Curve Analysis using traditional statistical approach

Using traditional statistical analysis, we aimed to evaluate the performance of SAPS II score at ICU admission in predicting HAIs for all patients staying in ICU for more than two days. Figure 2 shows the ROC curve with an AUC of $0.612(95 \%$ Confidence Interval $=0.60-0.63 ; p<0.001)$. Although this test was statistically significant, the accuracy of SAPS II score for predicting the risk of HAIs was of $56 \%$.

\section{ROC Curve Analysis using SVM model}

To improve the accuracy for predicting the risk of HAIs, we employed the SVM algorithm, working on SAPS II score along with other characteristics at ICU admission. Figure 3 shows the ROC curve of SVM prediction model for the test set. We report that the accuracy of the SVM classifier was $88 \%$ on the test set. Specifically, precision and recall were 0.95 and 0.91 for non-infected patients and 0.60 and 0.73 for those who were diagnosed with at least one HAIs during their ICU stay. In line, the predictivity was assessed using ROC curve, which provided an AUC of 0.90 (95\% Confidence Interval $=0.88-0.91 ; p<0.001)$. Our results indicated the reliability of our SVM- model against overfitting. Finally, we aimed to compare our prediction performance with those obtained on the same SVM model, without accounting for the SAPS II score variable in the test set. Figure 4 shows the ROC curve of SVM prediction model for the test set, reporting an accuracy of 78\%. Accordingly, precision and recall were 0.87 and 0.87 for non-infected patients and 0.31 and 0.32 for those infected, respectively. As expected, the AUC value provided by the ROC curve was $0.66(95 \%$ Confidence Interval $=0.65$ $0.68 ; p<0.001$ ), indicating a lower predictive ability.

\section{Discussion}

Identifying patients at higher risk of HAIs still represents a major challenge for public health, suggesting the need for novel tools that can guide patient management in ICUs [25-27]. To the best of our knowledge, the present study is the first one employing machine learning methods to identify patients at risk of HAIs, according to their individual characteristics at ICU admission. Indeed, there is current consensus that machine learning algorithms could support and enrich conventional statistical approaches, especially in terms of prediction of ICU prognosis, clinical deterioration and risk assessment [22, 23, 29]. Several modifiable and non-modifiable risk factors might affect the risk of HAIs and related adverse outcomes [4]. For instance, the prolonged use of invasive devices, patients' impaired immunity, surgical intervention and comorbidity represent the major risk factors for HAIs in ICU $[4,34]$.

In clinical practice, several prognostic scores are routinely used to evaluate the complex clinical-pathological conditions of ICU patients, in order to develop novel and more suitable preventive strategies tailored to each patient's requirements $[35,36]$. For instance, the SAPS II score represents the most useful tool for the prediction of prognosis, HAIs risk, sepsis and mortality [9-11, 13, 35].

To this aim, we first evaluated the ability of SAPS II score at ICU admission for predicting HAIs risk of 7827 patients staying in ICU for more than two days. Interestingly, our ROC curve analysis, which provides an AUC value of 0.612, does not suggest the use of SAPS II score in the end-of-life decision-making. Indeed, although the test was statistically significant, the accuracy of SAPS II score for predicting the risk of HAIs was of $56 \%$. In this scenario, machine learning approaches represent a possible strategy for healthcare facilities, making possible to build a specific prediction model targeted to demographics and clinical characteristics of patients $[22,23]$. In line, several studies suggested SVM technique as being an excellent and powerful algorithm to 
predict common complex diseases with many risk factors, having a better discrimination than conventional statistical approaches [37].

Accordingly, we employed the SVM algorithm, considering SAPS II score along with other characteristics at ICU admission (i.e. age, sex, SAPS II score at admission, patient's origin, type of admission, trauma, impaired immunity, non-surgical treatment for acute coronary disease, surgical intervention, presence of invasive devices, and antibiotic therapy), in order to improve the accuracy for predicting the risk of HAIs. Our findings demonstrated that the accuracy of the SVM classifier was $88 \%$ on the test set, reporting precision and recall values of 0.95 and 0.91 for non-infected patients and 0.60 and 0.73 for those who were diagnosed with at least one HAIs during their ICU stay. In line, the predictive ability assessed by the ROC curve provided an AUC of 0.90 .

To assess the relevance of patients' characteristic at ICU admission in our SVM model, we compared the prediction performance with those obtained by same SVM model, without accounting for the SAPS II score. We found a ROC curve reporting an accuracy of $78 \%$, with precision and recall values of 0.87 and 0.87 for noninfected patients and 0.31 and 0.32 for those infected, respectively. Notably, the AUC value provided by the ROC curve was of 0.66 , indicating a lower predictive ability. Due to its low predictive ability, our findings not warrant clinical usefulness of SAPS II score when considered alone, suggesting the need of an integrated approach with patients' personal and clinical characteristics, which are crucial in determining the risk of HAIs and adverse outcomes in ICU.

In conclusion, our findings provide a promising evaluation of a better predictive performance of the SVM algorithm than conventional statistical approaches, suggesting the SVM as a possible medical tool for a quickly patients management at ICU admission. Although further efforts are needed, predictive models in healthcare systems represent a useful strategy for better diagnosis, prognosis and personalized patients' management, including preventive strategies against HAIs [29].

\section{Figure legends}

Fig. 1 ROC curve of the SAPS II score predicting healthcare associated infections

Fig. 2 ROC curve of support vector machine algorithm predicting healthcare associated infections

Fig. 3 ROC curve of support vector machine algorithm predicting healthcare associated infections, by excluding SAPS II score 
Table 1. Characteristics of patients according to their infectious status

\begin{tabular}{|c|c|c|c|c|}
\hline Characteristics & $\begin{array}{l}\text { Patients } \\
(n=7827)\end{array}$ & $\begin{array}{l}\text { Infected patients } \\
(n=1225)\end{array}$ & $\begin{array}{l}\text { Non- infected } \\
\text { patients } \\
(n=6602)\end{array}$ & $p$-value \\
\hline Age, years & $69.0(21.0)$ & $69.0(21.0)$ & $69.0(21.0)$ & 0.064 \\
\hline Sex (\% men) & $60.6 \%$ & $62.8 \%$ & $60.1 \%$ & 0.084 \\
\hline \multicolumn{5}{|l|}{ Patient's origin } \\
\hline Other ward/healthcare facility & $73.9 \%$ & $67.7 \%$ & $75.1 \%$ & \multirow{3}{*}{$\begin{array}{l}<0.001 \\
<0.001\end{array}$} \\
\hline Community & $26.1 \%$ & $32.3 \%$ & $24.9 \%$ & \\
\hline SAPS II score at admission & $40.0(28.0)$ & $47.0(27.0)$ & $38.0(27.0)$ & \\
\hline \multicolumn{5}{|l|}{ Type of ICU admission } \\
\hline Medical & $47.6 \%$ & $53.6 \%$ & $46.5 \%$ & \multirow{2}{*}{$<0.001$} \\
\hline Surgical & $52.4 \%$ & $46.4 \%$ & $53.5 \%$ & \\
\hline Trauma & $3.4 \%$ & $5.0 \%$ & $3.2 \%$ & 0.001 \\
\hline Impaired immunity & $8.6 \%$ & $10.4 \%$ & $8.2 \%$ & 0.015 \\
\hline $\begin{array}{l}\text { Non-surgical treatment for acute } \\
\text { coronary disease }\end{array}$ & $10.2 \%$ & $8.9 \%$ & $10.4 \%$ & 0.109 \\
\hline Surgical intervention & $40.9 \%$ & $36.7 \%$ & $41.7 \%$ & $<\mathbf{0 . 0 0 1}$ \\
\hline $\begin{array}{l}\text { Antibiotic therapy in } 48 \text { hours } \\
\text { before ICU admission }\end{array}$ & $59 \%$ & $59.8 \%$ & $58.9 \%$ & 0.579 \\
\hline $\begin{array}{l}\text { Presence of urinary catheter at } \\
\text { ICU admission }\end{array}$ & $77.5 \%$ & $74.4 \%$ & $78.0 \%$ & 0.006 \\
\hline $\begin{array}{l}\text { Presence of intubation at ICU } \\
\text { admission }\end{array}$ & $59.8 \%$ & $63.8 \%$ & $59.1 \%$ & 0.002 \\
\hline $\begin{array}{l}\text { Presence of central venous } \\
\text { catheter at ICU admission }\end{array}$ & $41 \%$ & $39.7 \%$ & $41.3 \%$ & 0.295 \\
\hline ICU-acquired sepsis (\%yes) & $6.1 \%$ & $37.6 \%$ & - & - \\
\hline Outcome (\%death) & $23.2 \%$ & $35.1 \%$ & $21.0 \%$ & $<0.001$ \\
\hline Length of ICU stay, days & $5.0(10.0)$ & $20.0(20.0)$ & $4.0(6.0)$ & $<\mathbf{0 . 0 0 1}$ \\
\hline
\end{tabular}

*Results are reported as median (interquartile range) for continuous variables, or percentage for categorical variables.

Statistical analyses were performed using the Mann-Whitney or the Chi-squared test.

\section{Declarations}

Funding (information that explains whether and by whom the research was supported)

Conflicts of interest/Competing interests: The authors declare no conflict of interest

Ethics approval (include appropriate approvals or waivers)

Consent to participate (include appropriate statements)

Consent for publication (include appropriate statements)

Availability of data and material (data transparency)

Acknowledgements

The Authors wish to thank all colleagues, physicians and nurses in the participating hospitals and ICUs for their collaboration and for providing surveillance data. 


\section{\#Collaborators of the SPIN-UTI network:}

Murgia Paola, Maria Dolores Masia (Ospedale SS. Annunziata, Sassari); Brusaferro Silvio, Daniele Celotto, Arnoldo Luca (Azienda Sanitaria Universitaria Integrata, Udine); Bissolo Emanuela, Rigo Alberto (Azienda ULSS 21 di Legnago, Varese); Tardivo Stefano, Francesca Moretti, Alberto Carli (Azienda Ospedaliera Universitaria Integrata di Verona); Pascu Diana, Tessari Lorella (ULSS 20 di Verona; Ospedale di San Bonifacio, Verona); Bernasconi Mara Olga, Brusaferro Marco (Azienda ULSS 18, Rovigo); Pappalardo Federico (Università Vita-Salute San Raffaele, Milano); Francesco Auxilia (Università degli Studi di Milano; Azienda Ospedaliera Ospedale Niguarda Ca' Granda, Milano; Fondazione OMPMaRE, Presidio Ospedale Maggiore Policlinico, Milano); Fenaroli Salesia (Istituto Clinico Humanitas, Milano); Cesira Pasquarella (Università di Parma, Azienda USL di Parma, Ospedale di Fidenza); Sicoli Ennio (Azienda Ospedaliera, Cosenza); Montagna Maria Teresa (Azienda Ospedaliero Universitaria Consorziale Policlinico, Bari; Azienda Sanitaria Locale Ospedale "Di Venere", Bari); Egitto Giovanni, Squeri Raffaele (Azienda Ospedaliera Universitaria "G. Martino", Messina); Salvatore Tribastoni, Alessandro Pulvirenti, Sebastiano Catalano, Pietro Battaglia (Centro Clinico Diagnostico CCD G.B. Morgagni Catania" - Policlinico Morgagni, Catania); Bellocchi Patrizia, Castiglione Giacomo, Mattaliano Anna Rita, Astuto Marinella, La Camera Giuseppa (Azienda Ospedaliera Universitaria Policlinico Vittorio Emanuele, Catania); Longhitano Anna Maria, Giorgio Scrofani, Maria Concetta Monea (Azienda Ospedaliera Cannizzaro, Catania); Milazzo Marina (Presidio Ospedaliero di Vittoria, Ragusa; Presidio Ospedaliero Civile-OMPA, Ragusa; ASP 7, Ragusa); Giarratano Antonino, Calamusa Giuseppe, Torregrossa Maria Valeria (Azienda Ospedaliera Universitaria Policlinico Paolo Giaccone, Palermo); Di Benedetto Antonino (ASP di Palermo); Rizzo Giuseppa Maria Gisella (ASP di Trapani); Manta Giuseppe (ASP di Caltanissetta; Presidio Ospedaliero Sant'Elia, Caltanissetta); Tetamo Romano, Mancuso Rosa (ARNAS "Civico-Ascoli-Di Cristina", Palermo); Mella Laura Maria (Azienda Ospedaliera "Luigi Sacco", Milano); Dei Ignazio (ASL Lanusei, Presidio Ospedaliero "Nostra Signora della Mercede", Nuoro); Pandiani Irene (Presidio Ospedaliero SS. Annunziata, Taranto); Cannistrà Antonino, Piotti Paola (Clinica S. Rocco di Franciacorta S.p.A. Ome, Brescia); Girardis Massimo, Righi Elena, Barbieri Alberto (Policlinico di Modena); Crollari Patricia (Presidio Ospedaliero di Vizzolo Predabissi, Milano); Borracino Albino, Coniglio Salvatore, Palermo Rosaria, Pintaudi Sergio, Di Stefano Daniela (ARNAS Garibaldi, Catania); Romeo Antonina (Azienda Ospedaliera Villa Sofia-Cervello, Presidio Ospedaliero Villa Sofia, Palermo); Sticca Giovanna (Fondazione di Ricerca e Cura "Giovanni Paolo II" UCSC, Campobasso); Minerva Massimo (Azienda Ospedaliera di Melegnano - Presidio Ospedaliero di Cernusco sul Naviglio, Milano); Fabiani Leila, Gentile Alessandra (Presidio Ospedaliero S. Salvatore, L’Aquila); Stefanini Paolo (AUSL di Reggio Emilia; Ospedale Civile Di Guastalla, Reggio Emilia); D'Errico Marcello Mario, Donati Abele (Azienda Ospedaliero-Universitaria Ospedali Riuniti, Ancona); De Remigis Santa, Venturoni Federica (Presidio Ospedaliero di Teramo); Antoci Manuela (Presidio Ospedaliero Civile-OMPA, Ragusa; ASP 7, Ragusa); Pagliarulo Riccardo (Azienda Sanitaria Locale Ospedale "Di Venere", Bari); Bianco Aida, Pavia Maria (Azienda Ospedaliera Universitaria “Mater Domini”, Catanzaro); Pasculli Marcello, Vittori Cesare (Azienda Ospedaliera Universitaria Senese, Siena); Orsi Giovanni Battista (Azienda Ospedaliera Sant'Andrea, Roma); Arrigoni Cristina (IRCCS policlinico San Matteo di Pavia); Olori Maria Patrizia (Stabilimento Ospedaliero “C. e G. Mazzoni”, Ascoli Piceno); Antonelli Massimo, Laurenti Patrizia (Fondazione Policlinico Gemelli, Roma); Ingala Franco, Carmela Conte, Russo Salvatore, Condorelli Laura (ASP 8, Siracusa); Farruggia Patrizia (Presidio Ospedaliero Unico Aziendale dell'Azienda USL di Bologna); Cristina Maria Luisa (Ente Ospedaliero Ospedali Galliera di Genova); Galassi Italia (Presidio Ospedaliero "SS. Filippo e Nicola” di Avezzano). 


\section{References}

1. Alp E, Damani N, (2015) Healthcare-associated infections in intensive care units: epidemiology and infection control in low-to-middle income countries. J Infect Dev Ctries 9: 1040-1045

2. Haque M, Sartelli M, McKimm J, Abu Bakar M, (2018) Health care-associated infections an overview. Infect Drug Resist 11: 2321-2333

3. Serra-Burriel M, Keys M, Campillo-Artero C, Agodi A, Barchitta M, Gikas A, Palos C, López-Casasnovas G, (2020) Impact of multi-drug resistant bacteria on economic and clinical outcomes of healthcare-associated infections in adults: Systematic review and metaanalysis. PLoS One 15: e0227139

4. Marcel JP, Alfa M, Baquero F, Etienne J, Goossens H, Harbarth S, Hryniewicz W, Jarvis W, Kaku M, Leclercq R, Levy S, Mazel D, Nercelles P, Perl T, Pittet D, Vandenbroucke-Grauls C, Woodford N, Jarlier V, (2008) Healthcare-associated infections: think globally, act locally. Clin Microbiol Infect 14: 895-907

5. Suetens C, Latour K, Kärki T, Ricchizzi E, Kinross P, Moro ML, Jans B, Hopkins S, Hansen S, Lyytikäinen O, Reilly J, Deptula A, Zingg W, Plachouras D, Monnet DL, Group TH-AIPS, (2018) Prevalence of healthcare-associated infections, estimated incidence and composite antimicrobial resistance index in acute care hospitals and long-term care facilities: results from two European point prevalence surveys, 2016 to 2017. Euro Surveill 23

6. Agodi A, Barchitta M, Quattrocchi A, Maugeri A, Aldisio E, Marchese AE, Mattaliano AR, Tsakris A, (2015) Antibiotic trends of Klebsiella pneumoniae and Acinetobacter baumannii resistance indicators in an intensive care unit of Southern Italy, 2008-2013. Antimicrob Resist Infect Control 4: 43

7. European Centre for Disease Prevention and Control (2017) Healthcare-associated infections acquired in intensive care units. Annual Epidemiological Report for 2017.

8. European Centre for Disease Prevention and Control Point prevalence survey of healthcareassociated infections and antimicrobial use in European acute care hospitals 2011-2012.

9. Gilani MT, Razavi M, Azad AM, (2014) A comparison of Simplified Acute Physiology Score II, Acute Physiology and Chronic Health Evaluation II and Acute Physiology and Chronic Health Evaluation III scoring system in predicting mortality and length of stay at surgical intensive care unit. Niger Med J 55: 144-147

10. Sadaka F, EthmaneAbouElMaali C, Cytron MA, Fowler K, Javaux VM, O'Brien J, (2017) Predicting Mortality of Patients With Sepsis: A Comparison of APACHE II and APACHE III Scoring Systems. J Clin Med Res 9: 907-910

11. Mungan Ib, Bektaş Se, Çavuş MA, Sarı S, Turan S (2019) The predictive power of SAPS-3 and SOFA scores and their relations with patients outcomes in the Surgical Intensive Care Unit. In: Editor (ed)^(eds) Book The predictive power of SAPS-3 and SOFA scores and their relations with patients outcomes in the Surgical Intensive Care Unit. City, pp.

12. Haddadi A, Ledmani M, Gainier M, Hubert H, Tagne J, De Micheaux P Comparing the APACHE II, SOFA, LOD, and SAPS II scores in patients who have developed a nosocomial infection. In: Editor (ed)^(eds) Book Comparing the APACHE II, SOFA, LOD, and SAPS II scores in patients who have developed a nosocomial infection. City, pp.

13. Agodi A, Barchitta M, Auxilia F, Brusaferro S, D'Errico MM, Montagna MT, Pasquarella C, Tardivo S, Arrigoni C, Fabiani L, Laurenti P, Mattaliano AR, Orsi GB, Squeri R, 
Torregrossa MV, Mura I, Collaborators, (2018) Epidemiology of intensive care unitacquired sepsis in Italy: results of the SPIN-UTI network. Ann Ig 30: 15-21

14. Allyn J, Ferdynus C, Bohrer M, Dalban C, Valance D, Allou N, (2016) Simplified Acute Physiology Score II as Predictor of Mortality in Intensive Care Units: A Decision Curve Analysis. PLoS One 11: e0164828

15. Nielsen A, Thorsen-Meyer H, Belling K, Nielsen A, Thomas C, Chmura P, Lademann M, Moseley P, Heimann M, Dybdahl L, Spangsege L, Hulsen P, Perner A, Brunak S Survival prediction in intensive-care units based on aggregation of long-term disease history and acute physiology: a retrospective study of the Danish National Patient Registry and electronic patient records. In: Editor (ed)^(eds) Book Survival prediction in intensive-care units based on aggregation of long-term disease history and acute physiology: a retrospective study of the Danish National Patient Registry and electronic patient records. City, pp.

16. Agodi A, Barchitta M, Quattrocchi A, Spera E, Gallo G, Auxilia F, Brusaferro S, D'Errico MM, Montagna MT, Pasquarella C, Tardivo S, Mura I, group G-Sw, (2017) Preventable proportion of intubation-associated pneumonia: Role of adherence to a care bundle. PLoS One 12: e0181170

17. Agodi A, Auxilia F, Barchitta M, Brusaferro S, D'Errico MM, Montagna MT, Pasquarella C, Tardivo S, Mura I, SPIN-UTI network of the GISIOWorking Group of the Italian Society of Hygiene PeMaPHS, (2015) Antibiotic consumption and resistance: results of the SPINUTI project of the GISIO-SItI. Epidemiol Prev 39: 94-98

18. Agodi A, Auxilia F, Barchitta M, Brusaferro S, D'Alessandro D, Grillo OC, Montagna MT, Pasquarella C, Righi E, Tardivo S, Torregrossa V, Mura I, GISIO-SITI, (2013) Trends, risk factors and outcomes of healthcare-associated infections within the Italian network SPINUTI. J Hosp Infect 84: 52-58

19. Agodi A, Auxilia F, Barchitta M, Brusaferro S, D'Alessandro D, Montagna MT, Orsi GB, Pasquarella C, Torregrossa V, Suetens C, Mura I, GISIO, (2010) Building a benchmark through active surveillance of intensive care unit-acquired infections: the Italian network SPIN-UTI. J Hosp Infect 74: 258-265

20. Agodi A, Auxilia F, Barchitta M, D'Errico MM, Montagna MT, Pasquarella C, Tardivo S, Mura I, GISIO-SItI, (2014) [Control of intubator associated pneumonia in intensive care unit: results of the GISIO-SItI SPIN-UTI Project]. Epidemiol Prev 38: 51-56

21. Agodi A, Barchitta M, Mura I, Pasquarella C, Torregrossa MV, SItI G, (2018) The commitment of the GISIO-SItI to contrast Healthcare-Associated Infections and the experience of prevalence studies in Sicily. Ann Ig 30: 38-47

22. Komorowski M, (2019) Artificial intelligence in intensive care: are we there yet? Intensive Care Med 45: 1298-1300

23. Rajkomar A, Dean J, Kohane I, (2019) Machine Learning in Medicine. Reply. N Engl J Med 380: 2589-2590

24. Favara G, Riela P, Maugeri A, Barchitta M, Gallo G, Agodi A (2019) Risk of Pneumonia and associated outcomes in Intensive Care Unit: an integrated approach of Visual and Cluster Analysis.

25. Yee CR, Narain NR, Akmaev VR, Vemulapalli V, (2019) A Data-Driven Approach to Predicting Septic Shock in the Intensive Care Unit. Biomed Inform Insights 11: 1178222619885147

26. Chen L, Dubrawski A, Wang D, Fiterau M, Guillame-Bert M, Bose E, Kaynar AM, Wallace DJ, Guttendorf J, Clermont G, Pinsky MR, Hravnak M, (2016) Using Supervised Machine Learning to Classify Real Alerts and Artifact in Online Multisignal Vital Sign Monitoring Data. Crit Care Med 44: e456-463 
27. Churpek MM, Yuen TC, Winslow C, Meltzer DO, Kattan MW, Edelson DP, (2016) Multicenter Comparison of Machine Learning Methods and Conventional Regression for Predicting Clinical Deterioration on the Wards. Crit Care Med 44: 368-374

28. Masia MD, Barchitta M, Liperi G, Cantù AP, Alliata E, Auxilia F, Torregrossa V, Mura I, Agodi A, (GISIO) ISGoHH, (2010) Validation of intensive care unit-acquired infection surveillance in the Italian SPIN-UTI network. J Hosp Infect 76: 139-142

29. Linnen DT, Escobar GJ, Hu X, Scruth E, Liu V, Stephens C, (2019) Statistical Modeling and Aggregate-Weighted Scoring Systems in Prediction of Mortality and ICU Transfer: A Systematic Review. J Hosp Med 14: 161-169

30. European Centre for Disease Prevention and Control (2015) European surveillance of healthcare-associated infections in intensive care units- HAI-Net ICU protocol- Protocol version 1.02 .

31. Cortes C, Vapnik V Support-Vector Networks. In: Editor (ed)^(eds) Book Support-Vector Networks. City, pp.

32. Martos-Benítez FD, Larrondo-Muguercia H, León-Pérez D, Rivero-López JC, OramaRequejo V, Martínez-Alfonso JL, (2020) Performance of three prognostic models in critically ill patients with cancer: a prospective study. Int J Clin Oncol

33. D'Arrigo G, Provenzano F, Torino C, Zoccali C, Tripepi G I test diagnostici e l'analisi della curva ROC. In: Editor (ed)^(eds) Book I test diagnostici e l'analisi della curva ROC. City, pp.

34. Tan X, Rolls K, Wiseman T, Betihavas V Risk factors for Healthcare Associated Infections (HAI) or sepsis in trauma patients : an integrative literature review. In: Editor (ed $)^{\wedge}(\mathrm{eds})$ Book Risk factors for Healthcare Associated Infections (HAI) or sepsis in trauma patients : an integrative literature review. City, pp.

35. Girou E, Pinsard M, Auriant I, Canonne M, (1996) Influence of the severity of illness measured by the Simplified Acute Physiology Score (SAPS) on occurrence of nosocomial infections in ICU patients. J Hosp Infect 34: 131-137

36. Parimbelli E, Marini S, Sacchi L, Bellazzi R, (2018) Patient similarity for precision medicine: A systematic review. J Biomed Inform 83: 87-96

37. Yu W, Liu T, Valdez R, Gwinn M, Khoury MJ, (2010) Application of support vector machine modeling for prediction of common diseases: the case of diabetes and pre-diabetes. BMC Med Inform Decis Mak 10: 16 